\title{
Land Cover Separability Analysis of MODIS Time Series Data using a combined Simple Harmonic Oscillator and a mean reverting stochastic process
}

\author{
T. L. Grobler, E. R. Ackrermann, J. C. Olivier, A. J. van Zyl, and W. Kleynhans
}

\begin{abstract}
It is proposed that the time series extracted from Moderate Resolution Imaging Spectroradiometer satellite data be modeled as a simple harmonic oscillator with additive colored noise. The colored noise is modeled with an Ornstein-Uhlenbeck process. The Fourier transform and maximum likelihood parameter estimation are used to estimate the harmonic and noise parameters of the Colored Simple Harmonic Oscillator. Two case studies in South Africa show that reliable class differentiation can be obtained between natural vegetation and settlement land cover types, when using the parameters of the Colored Simple Harmonic Oscillator as input features to a classifier. The two case studies were conducted in the Gauteng and Limpopo provinces of South Africa. In the case of the Gauteng case study we obtained an average $\kappa=0.86$ for single band classification, while standard harmonic features only achieved an average $\kappa=0.61$. In conclusion the results obtained from the Colored Simple Harmonic Oscillator approach outperformed standard harmonic features and the minimum distance classifier.
\end{abstract}

Index Terms-Ornstein-Uhlenbeck, temporal classification, MODIS and SHO.

\section{INTRODUCTION}

The classification of land cover is an important problem in general, and in South Africa it has particular importance because of mostly unplanned land cover change driven by settlement expansion and migration of people in the southern parts of Africa. In this paper we are particularly interested in the classification performance that can be achieved using remotely sensed satellite data derived from high temporal resolution data based on the Moderate Resolution Imaging Spectroradiometer (MODIS) satellite. When compared to Advanced Very High Resolution Radiometer (AVHRR) data MODIS exhibits enhanced spectral and radiometric resolution, wide geographical coverage and improved atmospheric corrections, while preserving the same temporal resolution [2].

T.L. Grobler is with the Department of Electrical, Electronic and Computer Engineering, University of Pretoria as well as the Defense, Peace, Safety and Security, CSIR, Pretoria, South Africa.

E.R. Ackermann is with the Computational and Applied Mathematics Department at Rice University, Houston, Texas

J.C. Olivier is with the School of Engineering, University of Tasmania, Hobart, Australia.

A.J. van Zyl is with the Department of Mathematics and Applied Mathematics, University of Pretoria, Pretoria, South Africa.

W. Kleynhans is with the Department of Electrical, Electronic and Computer Engineering, University of Pretoria as well as the Remote Sensing Research Unit, Meraka Institute, CSIR, Pretoria, South Africa.

Address correspondence to T.L. Grobler, Defense, Peace, Safety and Security, CSIR, Meiring Naude Road, Pretoria, South Africa. Email: tgrobler@csir.co.za. Telephone: +27 128412949.
It has a low spatial resolution (500 meters pixels) but has a high temporal resolution ( 8 day sampling interval for the MCD43A4 product). This makes the classification over time robust since single date reflectance values of different classes may be unseparable due to the fact that they share similar spectral reflectance characteristics over the short term [1], [2]. A good review of classification in remote sensing can be found in [3]. There are however classification approaches that are specifically used for the classification of time series that should be mentioned, including principal component analysis [4], [5] phenological metrics [6], Fourier analysis [7] and wavelet analysis [8].

Fourier (or spectral) analysis, on Normalized Difference Vegetation Index (NDVI) time series in particular, has been used extensively for land cover classification (see for example [7], [9]-[12]), and it has been shown that reliable class separation can be achieved even when considering only the mean and seasonal spectral components [7], [12].

The objective of this paper is to extend the Fourier classification approach by using a novel parsimonious parametric model of the MODIS time series. The parsimonious parametric model consists of harmonic and colored noise parameters. The Fourier transform (FT) is used to extract the mean and the seasonal harmonic parameters from the time series and maximum-likelihood parameter estimation is used to extract the volatility and mean reversion rate of the remaining noise. The noise (residual) of remote sensing time series are modeled as either white or colored depending on whether all the information carrying frequency components have been removed or not [13]. As we only remove the mean and seasonal component we will use an appropriate colored noise model to describe our residual. The benefit of the approach presented here over the standard Fourier transform technique is that the less important Fourier features that by themselves do not contribute that much to classification accuracy are condensed into two model parameters that do contribute significantly to classification accuracy.

To emphasize the benefits of the parsimonious model we will show that (for two case studies in South Africa) when using the estimated proposed model parameters as features for a classifier we obtain better separability (between vegetation and settlements) and classification results, when compared to the results of a classifier that uses standard Fourier features [7], [12] or temporal features [2]. Our proposed technique also outperforms the minimum distance classifier [14].

The paper is organized as follows. We describe the parsimo- 
nious model in Section II. Section II also includes an algorithm for estimating the parameters of our proposed model. In the remaining part of the paper we perform two case studies to indicate the usefulness of the proposed model parameters, and the last section presents the conclusions.

\section{Proposed Model}

The rationale behind modeling remotely sensed time series data is to extract phenological markers from the data and for this reason models are normally used on vegetation index data only. The models are used to enable noise reduction so that meaningful markers can be extracted from the data. However in this paper we will not restrict ourselves to vegetation index data only. We will use our model as an aid to perform a separability analysis between different classes and not only to extract phenological markers. A simple model that can be used to represent remote sensed satellite time series is a Simple Harmonic Oscillator (SHO) given by

$$
A \sin \left(2 \pi f_{s} t+\phi\right)+C
$$

where

$$
\{A, C\}
$$

are the harmonic features proposed by [7], [12]. Many other models have also been proposed as an improvement on an SHO [12], [13], [16]-[20]. In particular [20] modeled MODIS time series with a harmonic non-linear solution of a chaotic attractor

$$
A \sin \left(2 \pi f_{s} t+\phi+\alpha \cos \left(2 \pi f_{s} t+\xi\right)\right)+C
$$

and [12] modeled NDVI time series with a triply modulated cosine

$$
A(t) \sin \left(2 \pi f_{s} t+\phi(t)\right)+C(t) .
$$

However as was shown in [7], [12] an SHO as underlying noise free model is effective. In this paper we will represent the mean $C$ and seasonal component $A$ of MODIS data with an SHO and model the remaining noise and harmonic components with a mean reverting stochastic process.

The structure of this section is as follows. We present our proposed model in Section II-A and the algorithm to estimate the parameters of our model in Section II-C. We compare our model to the models in the literature in Section II-B. Since the parameters of our model can be used as features in classification we also present an alternative feature extraction method of the data in Section II-D.

\section{A. Colored Simple Harmonic Oscillator}

Assuming we have an observed MODIS pixel $\mathbf{x}_{c}(t)$ belonging to class $c \in \mathcal{C}$. With $\mathbf{x}_{c}(t)$ we mean the set of signals $\left\{x_{c}^{b}(t)\right\}_{b \in\{1 \cdots 8\}}$ shown in Fig. 1 (here $\mathbf{x}[i]$ is the discrete analogue of $\mathbf{x}(t)$ ), where $b$ represents the MODIS band (seven land bands or NDVI). The $c$ is omitted if we do not know to which class a MODIS pixel belongs. Each observed signal belonging to the same class is a sample path of a stochastic process $X_{c}^{b}(t)$. We can therefore model each MODIS class $c$ as a set of stochastic processes $\mathbf{X}_{c}(t)=\left\{X_{c}^{b}(t)\right\}_{b \in\{1 \cdots 8\}}$. Since $X_{c}^{b}(t)$ is a stochastic process we can assign an analytic single multispectral pixel
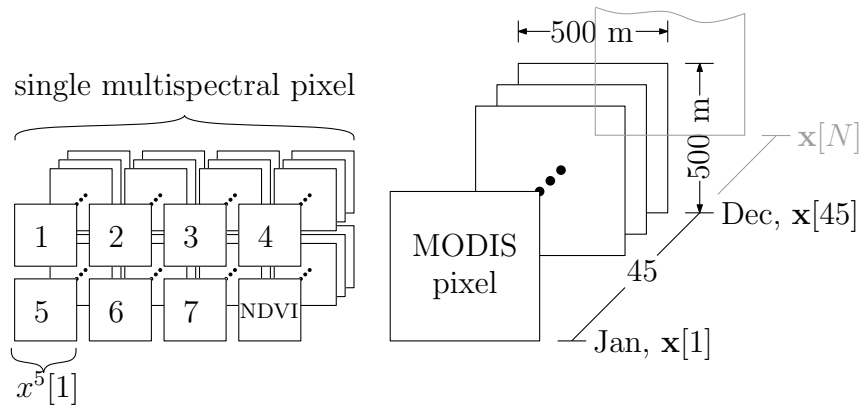

Fig. 1: Time series data representation for a single pixel.

expression (if such an expression exists) to each sample path (MODIS pixel) $x_{c}^{b}\left(t ; \boldsymbol{\theta}_{c}^{b}\right)$ of $X_{c}^{b}(t)$, where $\boldsymbol{\theta}_{c}^{b}$ is a set of random values with a joint probability density function. We thus have that $\boldsymbol{\theta}_{c}=\left\{\boldsymbol{\theta}_{c}^{b}\right\}_{b \in\{1 \cdots 8\}}$. For convenience we will sometimes omit $\boldsymbol{\theta}_{c}^{b}$ from $x_{c}^{b}\left(t ; \boldsymbol{\theta}_{c}^{b}\right)$.

We will see that the distribution of $\boldsymbol{\theta}_{c}^{b}$ is determined by the parameter set $\left\{A_{c}^{b}, \phi_{c}^{b}, C_{c}^{b}+\mu_{c}^{b}, \lambda_{c}^{b}, \sigma_{c}^{b}\right\}$. To reduce clutter, we will often omit the super- and subscripts $b$ and $c$.

The proposed analytic expression for each MODIS pixel (sample path) is given by

$$
x(t ; \boldsymbol{\theta})=s(t ;\{A, \phi, C\})+\eta(t ;\{\mu, \lambda, \sigma\}),
$$

where $s(t)$ is an SHO with period $T_{s}=\frac{1}{f_{s}}=45$ and equation

$$
s(t ;\{A, \phi, C\})=A \sin \left(2 \pi f_{s} t+\phi\right)+C .
$$

The noise process $\eta(t ;\{\mu, \lambda, \sigma\})$ is an Ornstein-Uhlenbeck process that satisfies the stochastic differential equation

$$
d \eta(t)=\lambda(\mu-\eta(t)) d t+\sigma d W(t) .
$$

Here $\mu \in \mathbb{R}$ is the long-term mean of the process, $\lambda>0$ is the rate of mean reversion, $\sigma>0$ is the volatility or average magnitude, per square-root time, of the random fluctuations, and $W(t)$ is a standard Brownian motion on $t \in[0, \infty)$. That is, $d W(t) \sim \mathcal{N}(0, \sqrt{d t})$. Of course, one should, for each class and band, expect $\mu$ to be insignificant compared to $C$, and to have $\mu=0$ if the parameter $C$ can be estimated without error.

It is important to notice that although (7) is a noise process, the mean reversion rate mainly models the remaining harmonic components (the remaining dependency after the subtraction of the SHO) of the underlying noise free signal, while the volatility parameter of (7) mostly models the actual noise added to the signal and the inter annual variation.

The Ornstein-Uhlenbeck process is widely used in mathematical finance for the modeling of the dynamics of interest rates and volatilities of asset prices. The Ornstein-Uhlenbeck process is the continuous-time analogue of the discrete time $\mathrm{AR}(1)$ process and, when initialised with the equilibrium distribution, is also stationary, Gaussian, Markov and mean reverting. The Ornstein-Uhlenbeck process can model a wider spectrum of noise types than just white noise.

The ensemble mean for $\mathbf{X}_{c}(t)$ is defined as

$$
\mathbf{y}_{c}(t)=\left\{\mathbb{E}\left[X_{c}^{b}(t)\right]\right\}_{b \in\{1 \cdots 8\}} .
$$




\section{B. Summary of Model Comparisons}

There are however some drawbacks to (3) and (4). Firstly fitting (3) to the data over a long period makes the model locally inaccurate, since the model does not compensate for annual variation. In other words (3) is useful only if we want to model each observed year separately, since continuous boundary conditions are not supported by Levenberg-Marquardt. Secondly the technique (Levenberg-Marquardt) used to estimate the parameters of (3) produces a local minimum if the initial estimate of the parameters is not close to their true values. Furthermore (3) can represent a wide variety of underlying noise free models, including period-halving bifurcations, but if this set of possible functions is extensive enough to model all noise free remotely sensed signals remains an open question. The model (4) is more general than (3), since the amplitude, mean and phase can be functions of time. The phase is also not restricted to a cosine. However a drawback of (4) is that it can not be described in a single parsimonious model equation, since $A, C$ and $\phi$ are functions of time. Lastly the noise process superimposed on the underlying noise free signal is not modeled by either (3) or (4) (or rather the noise is assumed to be white).

In contrast to the above the Colored Simple Harmonic Oscillator (CSHO) is a parsimonious model, where (7) not only models the noise (allows color) and remaining harmonic components, but to a certain extent also compensates for annual variation due to the volatility build into the OrnsteinUhlenbeck process. The CSHO model however does not explicitly try to represent the true underlying noise free model (not a smoothing technique). This is however not a problem, since we do not want to extract phenological markers from the data. Furthermore the assumption of an SHO as underlying noise free model is reasonable for the case studies presented in this paper, since the mean and seasonal harmonics in our data dominate the other harmonics significantly. Lastly (5) can also be used to create simulated data. Simulated data is useful for creating standardized testing platforms.

\section{Harmonic and Noise features}

To estimate the harmonic parameters of (5) we will use the Fourier transform, while the noise parameters will be estimated via maximum-likelihood parameter estimation. We define the Fourier transform of an observed MODIS pixel $\mathbf{x}(t)$ as

$$
\mathcal{X}(f)=\left\{\mathcal{F}\left[x^{b}(t)\right]\right\}_{b \in\{1 \cdots 8\}} .
$$

The subscript $c$ is omitted here, since we do not know to which class an observed pixel belongs.

For each band $b$ we can estimate $\{\hat{A}, \hat{\phi}, \hat{C}\}$ as follows

$$
\begin{aligned}
\hat{A} & =2\left|\mathcal{F}\left[x^{b}(t)\right]\left(f_{s}\right)\right| \\
\hat{\phi} & =\arg \left(\mathcal{F}\left[x^{b}(t)\right]\left(f_{s}\right)\right) \\
\hat{C} & =\left|\mathcal{F}\left[x^{b}(t)\right](0)\right|
\end{aligned}
$$

To estimate the parameters $\hat{\mu}, \hat{\lambda}$ and $\hat{\sigma}$ for $x^{b}(t)$ a maximum likelihood parameter estimation is used. We first calculate

$$
\hat{\eta}^{b}(t)=x^{b}(t)-\hat{A} \sin (2 \pi f t+\hat{\phi})+\hat{C} .
$$

Now let $\eta^{b}[i]$ be the discrete time analogue of $\eta^{b}(t)$, with $\delta$ being the time step of $\eta^{b}[i]$, i.e. $t=i \delta$, and $n$ being the total amount of samples we have of $\eta^{b}(t)$. The log-likelihood function of $\eta^{b}[i]$ is given by [15]

$$
\begin{aligned}
L(\mu, \lambda, \bar{\sigma}) & =-\frac{n}{2} \ln (2 \pi)-n \ln (\bar{\sigma})-\cdots \\
& \cdots \frac{1}{2 \bar{\sigma}^{2}} \sum_{i=1}^{n}\left[\eta^{b}[i]-\eta^{b}[i-1] \alpha-\mu(1-\alpha)\right]^{2},
\end{aligned}
$$

where

$$
\bar{\sigma}^{2}=\sigma^{2} \frac{1-e^{2 \alpha}}{2 \lambda}
$$

and

$$
\alpha=e^{-\lambda \delta}
$$

By respectively setting the partial derivative of (14) with respect to $\mu, \lambda, \bar{\sigma}$ equal to 0 and respectively solving for $\mu, \lambda, \bar{\sigma}$, such that $\mu$ is independent of $\lambda$ and $\bar{\sigma}$, we get the following maximum likelihood estimators

$$
\begin{gathered}
\hat{\mu}=\frac{\eta_{y} \eta_{x x}-\eta_{x} \eta_{x y}}{n\left(\eta_{x x}-\eta_{x y}\right)-\left(\eta_{x}^{2}-\eta_{x} \eta_{y}\right)}, \\
\hat{\lambda}=-\frac{1}{\delta} \ln \frac{\eta_{x y}-\hat{\mu} \eta_{x}-\hat{\mu} \eta_{y}+n \hat{\mu}^{2}}{\eta_{x x}-2 \hat{\mu} \eta_{x}+n \hat{\mu}^{2}}, \\
\hat{\bar{\sigma}}=\frac{1}{n}\left[\eta_{y y}-2 \hat{\alpha} \eta_{x y}+\hat{\alpha}^{2} \eta_{x x} \cdots\right. \\
\left.-2 \mu(1-\hat{\alpha})\left(\eta_{y}-\hat{\alpha} \eta_{x}\right)+n \mu^{2}(1-\hat{\alpha})^{2}\right],
\end{gathered}
$$

with

$$
\begin{gathered}
\eta_{x}=\sum_{i=1}^{n} \hat{\eta}^{b}[i-1], \eta_{y}=\sum_{i=1}^{n} \hat{\eta}^{b}[i] \\
\eta_{x x}=\sum_{i=1}^{n} \hat{\eta}^{b}[i-1]^{2}, \eta_{x y}=\sum_{i=1}^{n} \hat{\eta}^{b}[i-1] \hat{\eta}^{b}[i], \eta_{y y}=\sum_{i=1}^{n} \hat{\eta}^{b}[i]^{2},
\end{gathered}
$$

where the relation between $\sigma$ and $\bar{\sigma}$ was defined in (15) and $\alpha$ is given by (16). A resimulated example pixel reconstructed using (5) and the estimated parameters is given in Fig. 2. The

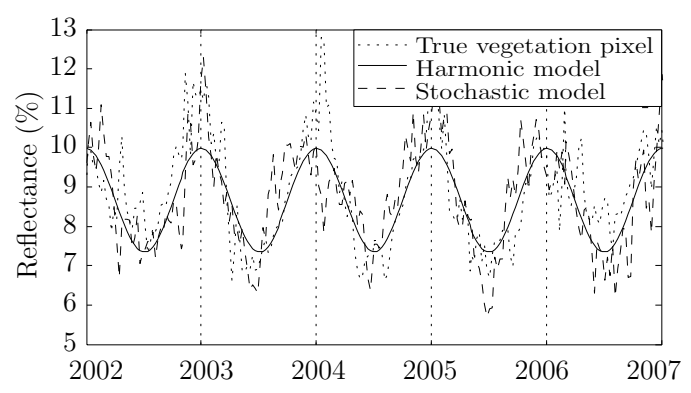

Fig. 2: A true and simulated MODIS vegetation pixel in spectral band four (Gauteng).

estimated parameters can now be used as input features of a classifier. 


\section{Temporal features}

Selecting temporal features for classification purposes is another well known approach [2]. If we want to choose the most relevant reflectance values (temporal features) from a MODIS pixel $\mathbf{x}(t)$ to use as features for a classifier, we need to select those reflectance values from $\mathbf{x}(t)$ where the annual ensemble mean of two different classes are at a maximum distance from each other. Mathematically we can write the following. We would like to select $\tau$ s.t. the following optimization problem is maximized.

$$
\sup _{\tau \in[0,45]}\left\|\overline{\mathbf{y}}_{c_{1}}(\tau)-\overline{\mathbf{y}}_{c_{2}}(\tau)\right\|_{2},
$$

where $\overline{\mathbf{y}}$ represents the annual ensemble mean. The solution $\tau$ can be extended to a sequence $\tau$, since the annual ensemble mean is periodic, we can thus obtain a maximum more than once during the observation period $T$. Now we select the actual reflectance values from the observed MODIS pixel, $\boldsymbol{\zeta}=\mathbf{x}(\boldsymbol{\tau})=\left\{x_{b}(\boldsymbol{\tau})\right\}_{b \in\{1 \cdots 8\}}$. We can of course construct a smaller $\boldsymbol{\zeta}$ from any subsets of $\overline{\mathbf{y}}_{c_{1}}(t), \overline{\mathbf{y}}_{c_{2}}(t)$ and $\mathbf{x}(t)$, as long as the subsets are constructed using the same spectral bands.

\section{Classes, Study AReas and Data Description}

The data used for the two case studies is discussed in detail in the following sections.

\section{A. Classes}

Two classes of land cover type, namely settlements and natural vegetation is considered in this paper. The most prevalent form of land cover change in South Africa is that of settlement expansion, driven by formal as well as informal new settlements, which is caused by migration of people in the southern parts of Africa [21]. As such, the detection or classification of the land cover in South Africa is an important issue, as natural vegetation is being converted into settlement on a continuing and often informal (unplanned) basis. In this study the settlements class contains pixels consisting of about $50 \%$ buildings, and 50\% vegetation, whereas the vegetation class contains pixels with more than $90 \%$ vegetation.

\section{B. Study Area}

Every pixel within each class has eight associated time series, with observations every eight days. The first seven time series correspond to the seven MODIS spectral land bands, while the 8th time series corresponds to NDVI. The eight time series extracted from a single pixel is shown in Fig. 1.

The time series data is derived from the MODIS MCD43A4 Bidirectional Reflectance Distribution Function (BRDF) corrected $500 \mathrm{~m}$ land surface reflectance product corresponding to a total area of approximately $230 \mathrm{~km}^{2}$ in Gauteng and $800 \mathrm{~km}^{2}$ in Limpopo, South Africa. The Gauteng and Limpopo provinces are shown in Fig. 3.

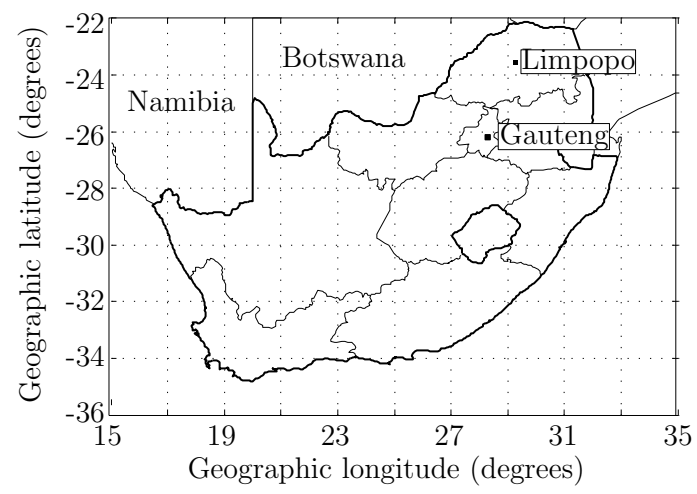

Fig. 3: Experimental study area: Gauteng and Limpopo, South Africa.

\section{Data Description}

The Gauteng dataset consists of 925 MODIS pixels, while the Limpopo data set contains 3232 MODIS pixels, identified by means of visual interpretation of high resolution Système Probatoire d'Observation de la Terre (SPOT) images between 2000 and 2008. Each pixel contains eight time series (seven MODIS bands, and NDVI), with $N=368$ observations. The Gauteng and Limpopo datasets are respectively divided into the two classes: settlements (333 Gauteng pixels and 1735 Limpopo pixels) and natural vegetation (592 Gauteng pixels and 1497 Limpopo pixels). The entire Gauteng data set and a subset of the Limpopo dataset were used in the studies [14] and [12] respectively.

\section{Separability Analysis: Gauteng Case Study}

We will investigate the separability of two classes in Gauteng by using a model in Section IV-B. Before we can perform our separability analysis we first need to verify that our data indeed fits the proposed model well, which is done in Section IV-A.

\section{A. Model Validation}

We know that the differences $\Delta \eta=\eta[t+1]-\eta[t]$ of the Ornstein-Uhlenbeck process are Gaussian. The question of how does the actual estimated noise $\hat{\eta}^{b}[t]$ (13) in Gauteng behave in comparison remains.

If we restrict our attention to an SHO, as the underlying noise free model, we find that the additive noise process is highly correlated. The differences $\Delta \hat{\eta}^{b}$ of the estimated noise process are however not Gaussian (the null hypothesis is rejected by the Kolmogorov-Smirnov test).

In fact, the $t$ location-scale distribution is a good fit. It has density function

$$
p(x)=\frac{\Gamma\left(\frac{\nu+1}{2}\right)}{\sigma \sqrt{\nu \pi} \Gamma\left(\frac{\nu}{2}\right)}\left[\frac{\nu+\left(\frac{x-\mu}{\sigma}\right)^{2}}{\nu}\right]^{-\left(\frac{\nu+1}{2}\right)},
$$

with location parameter $\mu$, scale parameter $\sigma>0$, and shape parameter $\nu>0$. The $t$ location-scale distribution is useful for modeling data distributions with heavier tails (more prone to 
outliers) than the normal distribution. It approaches the normal distribution as $\nu$ approaches infinity, and smaller values of $\nu$ yield heavier tails.

We argue however that the Ornstein-Uhlenbeck process is a good model fit for $\hat{\eta}^{b}[t]$, since we only sacrifice the capability to model the outliers effectively and in return gain a mathematical tractable model.

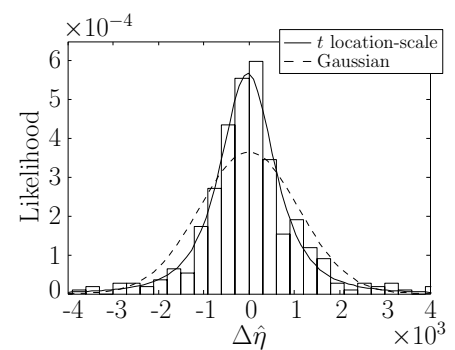

(a) Empirical (actual) increments.

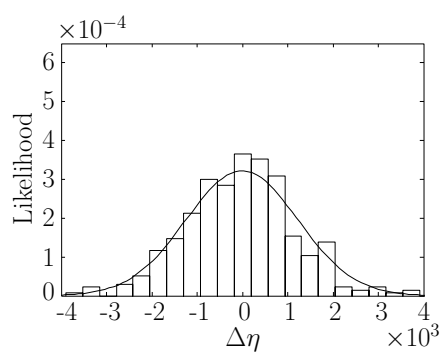

(b) Ornstein-Uhlenbeck increments.
Fig. 4: Increment distributions for a randomly selected vegetation pixel; $t$ location-scale and Gaussian approximations $(N=367)$. Derived using the recorded digital number.

In Fig. 4a we have the $t$ location-scale and Gaussian approximations of the increment distribution of a randomly selected vegetation pixel and in Fig. $4 \mathrm{~b}$ we have the increment distribution of an Ornstein-Uhlenbeck process.

\section{B. Ensemble Mean and Hellinger Distance}

Below we investigate the separability between the ensemble means of the classes as well as the estimated parameters of the classes.

1) Ensemble Mean: The ensemble mean for $\mathbf{X}_{c}(t)$ was defined in (8). The yearly ensemble mean for each class can be estimated by taking the daily average over all pixels and then over all years. In other words we assume inter annual variability and average it to obtain the yearly ensemble mean for each class for the period 2000 to 2008. The estimated yearly ensemble mean of both real world data and synthesized data generated using (5) show the same sinusoidal behavior with a period of one year (indicated in Fig. 5 and Fig. 6 for the real world data). We can now fit sinusoids through the data and assume this is the true value of $\overline{\mathbf{y}}_{c}(t)$.

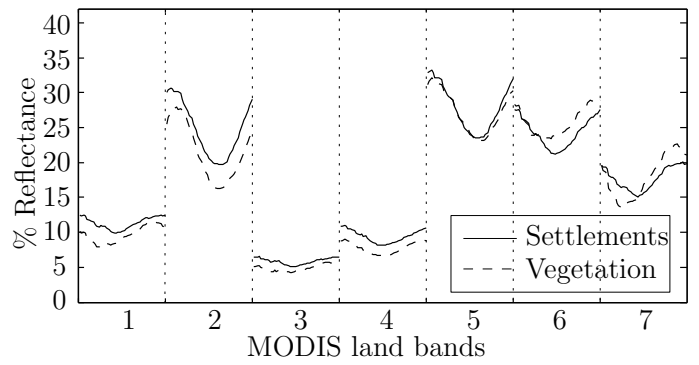

Fig. 5: The yearly ensemble mean of the MODIS land bands for the vegetation and settlement classes (Gauteng).

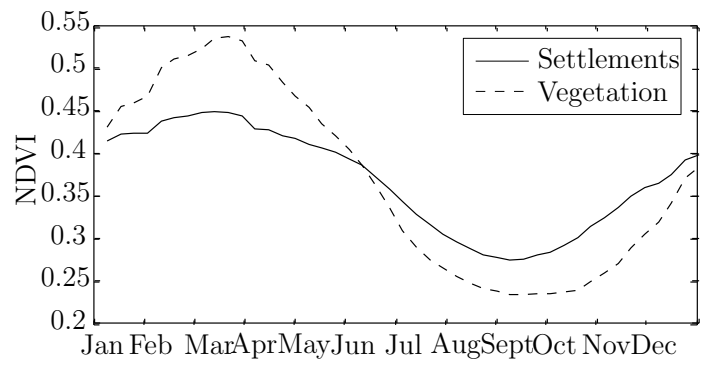

Fig. 6: The yearly ensemble mean of NDVI for the vegetation and settlement classes (Gauteng).

2) Hellinger distance: As discussed in Section II-C the parameters of $x^{b}(t)$ can be estimated. After estimation we can construct a probability density function for each parameter in each class using kernel density estimation. We can then calculate the Hellinger distance between the density functions of each parameter of the two classes. Recall that the Hellinger distance between probability density functions $p$ and $q$ is defined as

$$
H D(p, q)=\sqrt{1-\int_{-\infty}^{\infty} \sqrt{p(x) q(x)} d x} .
$$

A Hellinger distance of $H D(p, q) \approx 0$ indicates that the densities are not separable, whereas a distance $H D(p, q) \approx 1$ indicates that the densities are trivially separable. The calculated Helinger distances between the parameters of vegetation and settlements are given in Fig.7.

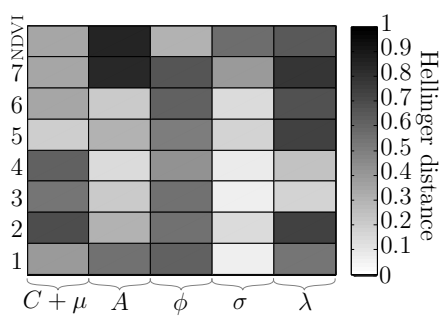

Fig. 7: Hellinger distance between the parameter probability density functions of the vegetation and settlements classes for each MODIS band (Gauteng).

3) Separability Discussion: From Fig. 5, Fig. 6 and Fig. 7 we can draw the following conclusions regarding vegetation and settlement separability. First we notice that vegetation and settlement pixels are separable by using only the mean component of (5) in bands 2 and 4. We also notice that vegetation and settlement pixels are trivially separable in bands 7 and NDVI when using the amplitude of (5). The phase parameter provides good separability in all the MODIS land bands. What is interesting from Fig. 7 is that we notice that the estimated noise parameters can also be used to separate vegetation and settlement classes. The mean reversion rate of (7) provides very good separability between settlements and vegetation classes in bands 2, 5, 7 and NDVI. It is noteworthy to mention that band 5 has very low separability except when using $\lambda$. Lastly the volatility $\sigma$ of (7) has a $H D(p, q) \approx 0$ 
in almost all of the MODIS bands, which implies that the vegetation and settlement classes are not that separable when using the parameter $\sigma$ by itself.

\section{Clasification Results: Gauteng Case Study}

We compare the classification results when using the parameters of the $\mathrm{CSHO}$ as features with the results obtained when using the proposed harmonic features (2) of [7], [12] and the temporal features [2] in Section II-D. We also compare the CSHO approach with the classification technique proposed in [14]. We are however not proposing a novel classification technique, instead we are using the classification results to validate the usefulness of the parameters of the proposed model. The proposed model only models no change pixels, but can be extended to model changed pixels as well. Furthermore change from vegetation to settlements when employing coarse resolution data is such a rare event that the assumption of no change is acceptable. A large amount of pixels will still be classified correctly under this assumption.

We start of by explaining the different classifiers used in Section V-A and Section V-B. We divide the classification results into two main sections. We will first discuss the classification results obtained by using up to two MODIS bands at a time in Section V-C. We then report our results of the remaining band combinations in Section V-D.

\section{A. Minimum Distance Classifier}

The minimum distance classifier [14] classifies the observed signal $\mathbf{x}(t)$ as class $c$ by choosing the class with the lowest model error. Where the model error for each class $c$ is defined as the accumulated euclidean distance between the observed signal $\mathbf{x}(t)$ and the signal model (yearly ensemble mean) $\overline{\mathbf{y}}_{c}(t)$. Mathematically we want to find a $c$ s.t. the following optimization problem is minimized

$$
\inf _{c \in \mathcal{C}} \int_{0}^{T}\left\|\mathbf{x}(t)-\overline{\mathbf{y}}_{c}(t)\right\|_{2} d t .
$$

Any subset of $\mathbf{x}(t)$ and $\overline{\mathbf{y}}_{c}(t)$ can be used for classification, as long as both subsets are constructed from the same spectral bands. The euclidean differences are normalized with the difference between the maximum and minimum observed value in each band.

\section{B. Support Vector Machine}

A Support Vector Machine (SVM) constructs a hyperplane or set of hyperplanes in a high or infinite dimensional space, which can be used for classification, regression, or other tasks [22]-[24]. We chose an SVM as classification technique since SVMs, unlike neural networks, are robust to the overfitting problem (increased spectral view increases feature set sizes). The first documented use of SVMs in remote sensing was in [25]. Since then there have been many important studies and results [26]-[29]. SVMs have also been applied to MODIS [2], [30]-[34]. A linear kernel with a $C$ selected via grid search was chosen. The linear kernel proved sufficient to validate the usefulness of the features of the CSHO. We used $50 \%$ of the pixels for training and $50 \%$ for validation.

\section{Two band classification results}

In Fig. 8a we have the $\kappa$ coefficients of every single band as well as every two band combination produced by the minimum distance classifier. In Fig. $8 b$ to Fig. $8 d$ we have the single and two band SVM classification results for different feature sets. The features used to produce the results in Fig. $8 \mathrm{~b}$ to Fig. 8d were temporal features, harmonic features and the parameters of the CSHO respectively. We can see that overall the parameters of the CSHO outperforms the other features and classification technique. By further inspection we also

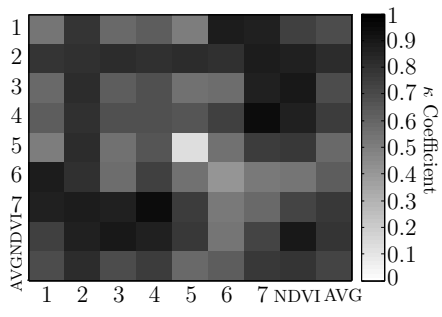

(a) Minimum distance classifier

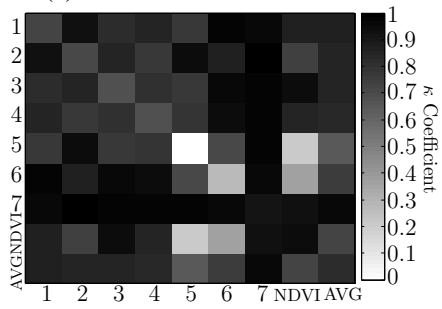

(c) Harmonic features [7], [12]

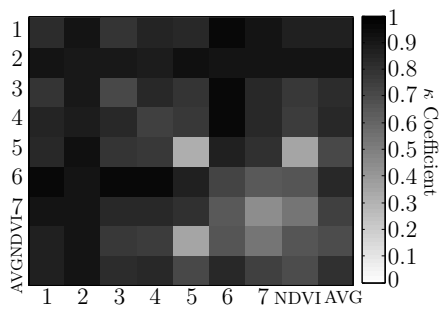

(b) Temporal features [2]

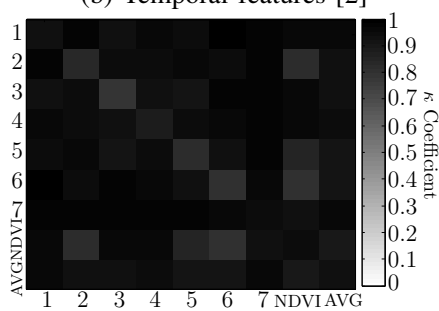

(d) Harmonic and noise features
Fig. 8: Two band classification results in Gauteng.

see that the best band to use in combination is band two in the case of Fig. 8a and Fig. 8b. However according to Fig. 8c and Fig. 8d the best band to use in combination is band seven. This difference can be explained with the aid of Fig. 5 and Fig. 7. The minimum distance classifier and the SVM with temporal input features will perform well if there exists a large average euclidean distance between the yearly ensemble means of the classes, while the remaining feature sets rely on the separability of the harmonic components for good classification results. As we can see from Fig. 5 the yearly ensemble mean is the most separable in band two, while according to Fig. 7 the seasonal component is the most separable in band seven, which explains the mentioned discrepancy. In general band five is the worst band to use in combination.

As an interesting side note we end our analysis by looking at the best and worst two band combination from Fig. 8a. The class models of the best and worst two band combination are displayed in Fig. 9b and Fig. 9a respectively.

It is clear from Fig. 9 that in the case of band four and seven the class models of settlement and vegetation are further apart than in band one and five.

\section{Multi band classification results}

Finally we present the graphs of the average kappa coefficients for each method in Fig. 10. For example if we restrict ourselves to two spectral bands we get 28 unique band 

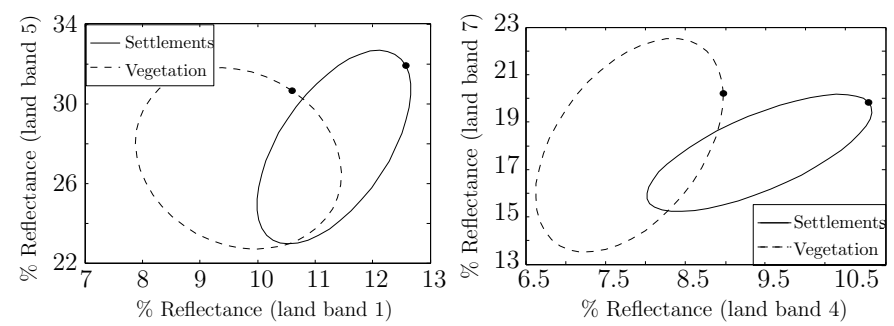

(a) Yearly ensemble mean models of (b) Yearly ensemble mean models of band 1 and 5 . band 4 and 7 .

Fig. 9: Two dimensional yearly ensemble mean models.

combinations. The $28 \kappa$ coefficients for all four methods are displayed in Fig. 8. The average of the $28 \kappa$ coefficients of each method form the four points in Fig. 10 each with an $x$ coordinate equal to 2. The most important result from Fig. 10 is that the average classification accuracy increases as one uses more spectral bands for classification. It is important to note here that even though the different methods perform on average similar when using a high number of spectral bands we still get a large improvement when a low number of spectral bands are used with the harmonic and noise feature set. The effectiveness of the harmonic and noise features validates our proposed model and is significant, since it is obviously more advantageous to classify more accurately without having to increase the spectral view.

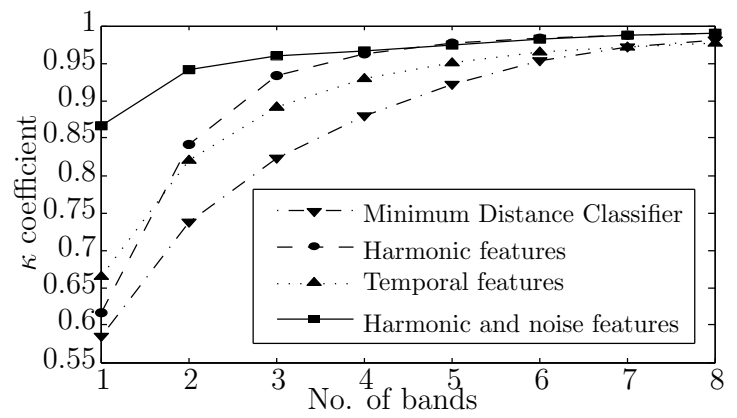

Fig. 10: Average $\kappa$ coefficients for all band combinations.

\section{Separablity Analysis: Limpopo Case Study}

The settlement class in Limpopo consists mostly of informal settlements. When we compare the informal settlement pixels in Limpopo to the formal settlement pixels in Gauteng we notice that informal settlements are less dense and for this reason contain a lot more residual vegetation. We discuss the separability between vegetation and settlements in Section VI-A.

\section{A. Ensemble Mean and Hellinger Distance}

1) Ensemble Mean: The estimated yearly ensemble means of the vegetation and settlement classes are given in Fig. 11 and Fig. 12. If we compare the Limpopo results to the Gauteng results we notice the same sinusoidal behavior. The differences between the yearly ensemble means of the two provinces can be ascribed to differences in indigenous vegetation of the two regions, geographical location and settlement density. We also notice that the ensemble means in Limpopo are more separable than in the case of Gauteng.

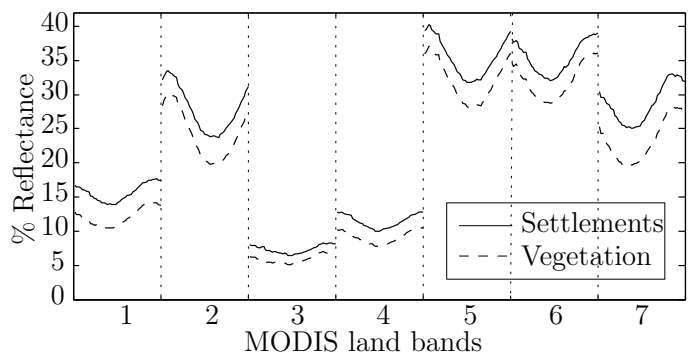

Fig. 11: The yearly ensemble mean of the MODIS land bands for the vegetation and settlement classes (Limpopo).

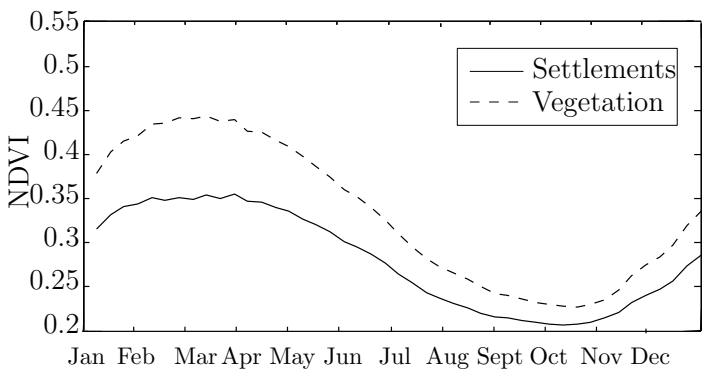

Fig. 12: The yearly ensemble mean of NDVI for the vegetation and settlement classes (Limpopo).

2) Hellinger Distance: The Hellinger distances between the different parameters of the CSHO model in Limpopo is displayed in Fig. 13. The Hellinger distances are much less than in the case of Gauteng. In other words the two classes in Limpopo is less separable than in Gauteng due to a high amount of residual vegetation in the settlement class. Even though the yearly ensemble means in Limpopo are more separable the two classes are actually less separable due to a high amount of inter class variation (high variance exist in the data).

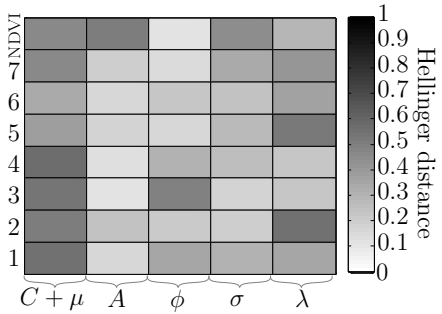

Fig. 13: Hellinger distance between the parameter probability density functions of the vegetation and settlements classes for each MODIS band (Limpopo).

3) Separability Discussion: According to Fig. 13, Fig. 11 and Fig. 12 the mean component contributes the most to the separability of the two classes, while the seasonal component contributes very little, except in the case of NDVI. However the most important result from Fig. 13 is that we can confirm that the noise parameters further enhance ones capability to discern between the two classes. 


\section{ViI. Clasification Results: Limpopo Case Study}

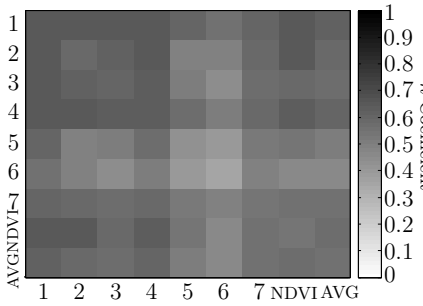

(a) Minimum distance classifier

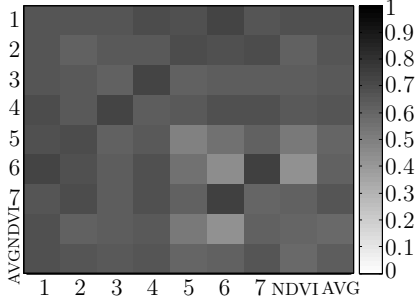

(c) Harmonic features [7], [12]

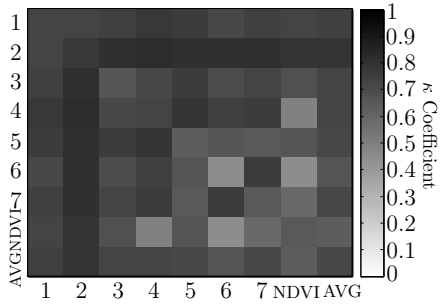

(b) Temporal features [2]

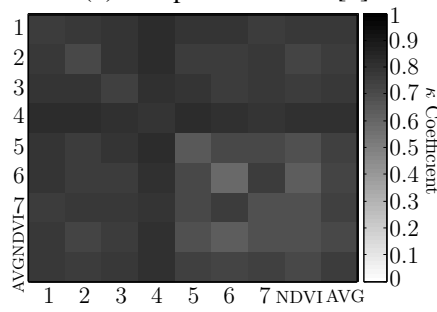

(d) Harmonic and noise features

Fig. 14: Two band classification results in Limpopo.

All the one band and two band classification results for the Limpopo case are given in Fig. 14. On average the harmonic an noise feature results are better than the remaining results in Fig. 14, supporting the usefulness of the proposed feature set. In Fig. 14d we see that the best band to use in combination is band four. It is important to note that this is not the best band predicted by Fig. 13. This discrepancy can be explained by realizing that a large Hellinger distance indicates that one should be able to find a hyperplane that provides good separability, but that this relation between large distance and good separability is not necessarily a perfect one to one relation. The weak performance of the minimum distance classifier in spite of highly separable yearly ensemble means confirms a high amount of variance in the data.

\section{EXTENDABILITY}

The focus of this paper is on the two classes namely vegetation and settlement, but the approach presented here is well suited to solve a multi class classification problem as well. The model itself can be applied to different classes, because it is quite general. It models the basic mean and seasonal components inherent in the remote sensing time series and then models the remaining residue with an appropriate color noise model. Furthermore it is well known that SVM's can solve multi class classification problems [2] and as such would be well suited for extending the approach to multiple classes. Lastly since we do not perform feature reduction on our model parameters we also do not have to perform preliminary class analysis before applying our algorithm to such problems.

\section{Conclusions}

To achieve class differentiation or accurate classification, we proposed a parsimonious model for the time series extracted from MODIS data for settlement and vegetation pixels. The model we proposed consisted of a harmonic and noise component whose parameters were estimated by using the Fourier

transform and maximum-likelihood parameter estimation respectively. Using two case studies we showed that when using the estimated proposed model parameters as features for a classifier we obtain better separability and classification results (between vegetation and settlements in Gauteng and Limpopo) if compared to the Fourier features [7], [12], temporal features [2] or the minimum distance classification technique in [14].

\section{ACKNOWLEDGMENTS}

The authors would like to thank Willem Marais of the Remote Sensing Research Unit (RSRU) at the CSIR, for his many comments and inputs. Alex Fortesque and Naledzani Mudau of CSIR, Satellite Application Centre (SAC) for providing the data on settlements. Research was funded by the CSIR Strategic Research Panel.

\section{REFERENCES}

[1] S.M. Davis et al., Remote Sensing: The Quantitative Approach, 1st ed. USA, McGraw-Hill, 1978.

[2] H. Carrão, P. Gonçalves, and M. Caetano, "Contribution of multispectral and multitemporal information from MODIS images to land cover classification," Remote Sensing of Environment, vol. 112, pp. 986-997, 2008.

[3] D. Lu and Q. Weng, "A survey of image classification methods and techniques for improving classification performance," International Journal of Remote Sensing, vol. 28, no. 5, pp. 823-870, 2007.

[4] R. Lasaponara,"Estimating inter annual variations in vegetated areas of Sardinia island using SPOT/VEGETATION NDVI temporal series," IEEE Geoscience and Remote Sensing Letters, vol. 3, no. 4, pp. 481-483, Oct. 2006.

[5] M. Hall-Beyer, "Comparison of single-year and multiyear NDVI time series principal components in cold temperate biomes.," IEEE Transactions on Geoscience and Remote Sensing, vol. 41, no. 11, pp. 2568-2574, Nov. 2003.

[6] D. Alcaraz, J. Paruelo, and J. Cabello,"Identification of current ecosystem functional types in the Iberian peninsula," Global Ecology Biogeography, vol. 15, no. 2, pp. 200-212, Mar. 2006.

[7] S. Lhermitte et al., "Hierachical image segmentation based on similarity of NDVI time-series," Remote Sensing of Environment, vol. 112, no. 2, pp. 506-512, Feb. 2008.

[8] G.L. Galford et. al., "Wavelet analysis of MODIS time series to detect expansion and intensification of row-crop agriculture in Brazil." Remote Sensing of Environment, vol. 112, pp. 576-587, 2008.

[9] R.I.N Juarez and W.T. Liu, "FFT analysis on NDVI annual cycle and climatic regionality in northeast Brasil," International Journal of Climatology, vol. 21, no. 14, pp. 1803-1820, Nov. 2001.

[10] B.P. Salmon et al.,"Automated land cover change detection: the quest for meaningful high temporal time series extraction," Proceedings of IEEE Geoscience and Remote Sensing Symposium, Honolulu, Hawaii, USA, July 2010, pp 1-4.

[11] B.P. Salmon et al.,"Unsupervised Land Cover Change Detection: Meaningful Sequential Time Series Analysis," IEEE Journal of Selected Topics in Applied Earth Observations and Remote Sensing, vol. 4 no. 2, pp. 327 335, Jun. 2011

[12] W. Kleynhans et al., "Improving land cover class seperation using an extended Kalman filter on MODIS NDVI Time-Series Data," IEEE Geoscience and Remote Sensing Letters, vol. 7, no. 2, pp. 381-385, 2010.

[13] B. Jiang et. al., "Modeling MODIS LAI time series using three statistical methods." Remote Sensing of Environment, vol. 114, pp. 1432-1444, 2010.

[14] E.R. Ackermann et al., "Minimum error land cover separability analysis and classification of MODIS time series data," Proceedings of IEEE Geoscience and Remote Sensing Symposium, Vancouver, Canada, July 2011.

[15] T. van den Berg, "Calibrating the Ornstein-Uhlenbeck model," SITMO, May 28 2011, [Online]. Available: http://www.sitmo.com/article/calibrating-the-ornstein-uhlenbeck-model/

[16] P. Jönsson and L. Eklundh, "Seasonality Extraction by Function Fitting to Time-Series of Satellite Sensor Data," IEEE Transactions on Geoscience and Remote Sensing, vol. 40, no. 8, pp. 1824-1832, Aug. 2002. 
[17] X. Zhang, M. A. Friedl, C. B. Schaaf, A. H. Strahler, J. C. F. Hodges, F. Gao, B. C. Reed, and A. Huete, "Monitoring vegetation phenology using MODIS," Remote Sensing of Environment, vol. 84, no. 3, pp. 471475, Mar. 2003.

[18] P. S. A. Beck, C. Atzberger, K. A. Høgda, B. Johansen, and A. K. Skidmore, "Improved monitoring of vegetation dynamics at very high latitudes: A new method using MODIS NDVI," Remote Sensing of Environment, vol. 100, no. 3, pp. 321-334, Feb. 2006.

[19] J. I. Fisher, J. F. Mustard, and M. A. Vadeboncoeur, "Green leaf phenology at Landsat resolution: Scaling from the field to the satellite," Remote Sensing of Environment, vol. 100, no. 2, pp. 265-279, Jan. 2006.

[20] H. Carrão, P. Gonçalves, and M. Caetano, "A nonlinear harmonic model for fitting satellite image time series: Analysis and prediction of land cover dynamics," IEEE Transactions on Geoscience and Remote Sensing, vol. 48, no. 4, pp. 1919-1930, 2010.

[21] B. P. Salmon et al., "The use of a multilayer perceptron for detecting new human settlements from a time series of MODIS images," International Journal of Applied Earth Observation and Geoinformation, vol. 13, no. 6, pp. 873-883, 2010

[22] V.N. Vapnik and A.Y. Chervonekis, "On the uniform convergence of relative frequencies of events to their probabilities," Theory of Probability and its applications, vol. 17, pp. 264-280, 1971.

[23] V.N. Vapnik, The Nature of Statistical Learning Theory, New York: Springer-Verlag, 1995.

[24] C.J.C. Burges, "A tutorial on Support Vector Machines for pattern recognition," Data Mining and Knowledge Discovery, vol. 2, pp. 121$167,1998$.

[25] J.A. Gualtieri and R.F. Cromp, "Support Vector Machines for hyperspectral remote sensing classification," Proceedings of the SPIE, 27th AIPR Workshop: Advances in Computer Assisted Recognition, Washington, DC, October 1998, pp. 221-232.

[26] C. Huang, L.S. Davis and J.R.G. Townshend, "An assessment of Support Vector Machines for land cover classification," International Journal of Remote Sensing, vol. 23, pp.725-749, 2002.

[27] G. Zhu and D.G. Blumberg, "Classification of ASTER data and SVM algorithms: The case study of Beer Sheva, Israel," Remote Sensing of Environment, vol. 80, no.2, pp.233-240, 2002.

[28] F. Melgani and L. Bruzzone, "Support Vector Machines for classification of hyperspectral remote-sensing images," Proceedings of IEEE Geoscience and Remote Sensing Symposium, Toronto, Canada, 2002, pp. 506-508, 2002.

[29] M. Pal and P.M. Mather, "Support Vector Machines for classification in remote sensing," International Journal of Remote Sensing, vol. 26, pp. 1007-1011, 2005.

[30] Y. Liu, Y. Xu, R. Shi and Z. Niu, "Evaluation of various classifiers on regional land cover classification using MODIS data," Proceedings of IEEE Geoscience and Remote Sensing Symposium, July 2005, pp. 12811283.

[31] C. Ye, Y. Liu, J. Peng, Ping Song and Dongbo Zhao, "Improving MODIS land cover classification using NDVI time-series and Support Vector Machine in the Poyang Lake Basin, China," Wireless Communications Networking and Mobile Computing, Chengdu, September 2010, pp. 1-4.

[32] H. Cai and S. Zhang, "Regional land cover classification from MODIS time-series and geographical data using support vetor machine ," 2010 IEEE Youth Conference on Information Computing and Telecommunications, Beijing, November 2010, pp. 102-105.

[33] J. Guo, J. Zhang, Y. Zhang and Y. Cao, "Study on the comparison of the land cover classification for multitemporal MODIS images," International Workshop on Earth Observation and Remote Sensing Applications, Beijing, June 2008, pp. 1-6.

[34] Shijin Li, Yuelong Zhu, Jun Feng, Ping Ai and Xi Chen, "Comparative Study of Three Feature Selection Methods for Regional Land Cover Classification Using MODIS Data," Congress on Image and Signal Processing, 2008, vol. 4, pp. 565-569.

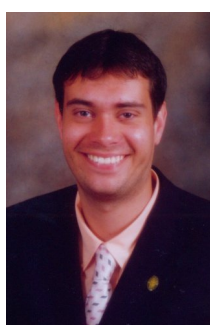

T.L. Grobler received his B.Eng. and M.Eng. degrees from the University of Pretoria, South Africa, in 2005 and 2008 respectively. He is currently a $\mathrm{Ph} . \mathrm{D}$. student at the University of Pretoria, South Africa. His research interests include remote sensing, time-series analysis, wireless communications, statistical detection and machine learning.

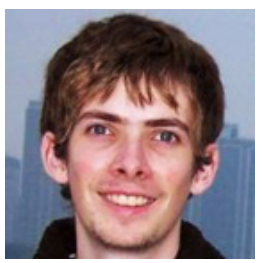

E.R. Ackermann is pursuing a Ph.D. in the Computational and Applied Mathematics department at Rice University. With the support of the 2011 International Fulbright Science and Technology Award, he is focusing on scientific computing and inverse problem theory. A native of South Africa, Etienne received a bachelors degree in computer engineering and a masters degree in electronic engineering; both from the University of Pretoria. His previous research experience comprised the development of an automatic facial detection and recognition system for security surveillance applications, the development of ionospheric total electron content models using Gaussian processes, and the statistical classification of coarse-resolution satellite images for environmental monitoring.

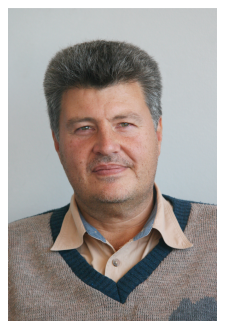

J.C. Olivier is a Professor of Engineering at the University of Tasmania in Australia. He was with Bell Northern Research in Ottawa Canada, Nokia Research Center in the United States, and the University of Pretoria in South Africa. He is an associate editor for the IEEE Transactions on Wireless Communication Letters and a past editor of the IEEE Trans. on Wireless Communications. His research interests are in the theory of estimation and detection applied to Remote Sensing and Communications theory.

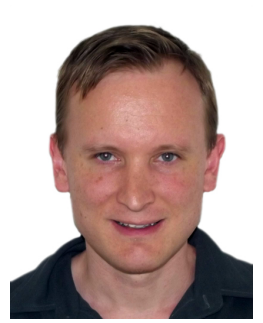

A.J. van Zyl was born in Pretoria, South Africa, on 13 June 1977. After his undergraduate studies, geared towards the actuarial qualification, he switched to mathematics. He obtained a Ph.D., with a thesis on tensor products of Banach spaces, in 2009. He is a lecturer at the Department of Mathematics and Applied Mathematics, University of Pretoria. His interests include Functional Analysis as well as the theory and applications of Stochastic Processes.

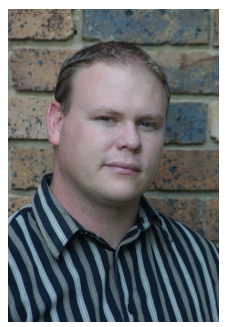

W. Kleynhans received the B.Eng. , M.Eng. and $\mathrm{Ph} . \mathrm{D}$. (Electronic Engineering) from the University of Pretoria, South Africa, in 2004, 2008 and 2011 respectively. He is currently a senior researcher with the Remote Sensing Research Unit at the Council for Scientific and Industrial Research in Pretoria, South Africa. His research interests include remote sensing, time-series analysis, wireless communications, statistical detection and estimation theory, and machine learning. 